\title{
The Retinoid Antagonist MX781 Induces Clusterin Expression in Prostate Cancer Cells via Heat Shock Factor-1 and Activator Protein-1 Transcription Factors
}

\author{
Yolanda Bayon, ${ }^{1}$ Maria A. Ortiz, ${ }^{1}$ Francisco J. Lopez-Hernandez, ${ }^{1}$ Philip H. Howe, ${ }^{2}$ and F. Javier Piedrafita ${ }^{1}$ \\ ${ }^{1}$ Sidney Kimmel Cancer Center, San Diego, California, and ${ }^{2}$ The Lerner Research Institute, Cleveland Clinic Foundation, Cleveland, Ohio
}

\section{ABSTRACT}

Retinoids mediate numerous biological responses through the transcriptional activation of nuclear retinoid receptors. Due to their antiproliferative activity, retinoids have shown promise as anticancer agents. Synthetic analogs have been described that selectively activate one subset of the retinoid receptors or inhibit their transcriptional activity. Some of these compounds exhibit strong anticancer activity, which is associated with their ability to induce apoptosis. Here we describe that the retinoid antagonist MX781 causes a substantial increase of clusterin mRNA and protein levels in prostate carcinoma cells. In contrast, retinoic acid and other synthetic agonists and antagonists show no effect on clusterin mRNA/protein levels. Induction of clusterin mRNA is associated with transcriptional activation of the clusterin promoter, which requires the proximal -218-bp region containing binding sites for heat shock factor (HSF)-1, activator protein (AP)-2, and AP-1 transcription factors. MX781 slightly induces AP-1 DNA binding activity, and mutation of the AP-1 site differentially affects the activation of the clusterin promoter in a cell type-specific manner. In contrast, a robust increase of HSF-1 DNA binding activity is observed in all cancer cell lines examined, and mutation of the heat shock element site in the clusterin promoter completely abolishes MX781-induced transcriptional activation in PC3 and DU145 cells. Other agonist retinoid-related molecules also induce AP-1 activity, but not HSF-1, and elicit no effect on clusterin expression levels. These data point to HSF-1 as an important factor regulating clusterin expression in response to MX781, although AP-1 activity may also participate in a cell type-specific manner.

\section{INTRODUCTION}

Retinoids are natural and synthetic derivatives of vitamin $\mathrm{A}$ that regulate gene expression by binding to and modulating the transcriptional activity of the nuclear retinoid receptors, retinoic acid receptors (RARs), and retinoid X receptors (reviewed in Ref. 1). Retinoids have been of particular interest in cancer prevention and treatment, but their broad activities mediated by transactivation of their receptors are often associated with secondary effects that have limited clinical applications. Selective analogs that target individual receptors or antagonize receptor activity are expected to display reduced toxicity. Synthetic retinoid-related molecules (RRMs), including the RAR $\gamma$ selective agonists (CD437, CD2325, and MX2870-1) and one RAR antagonist (MX781), have shown exceptional anticancer activity through the induction of apoptosis (see Refs. 2 and 3 and the references therein), which seems independent of the nuclear receptors (3, 4). Other RRMs of the heteroarotinoid class also induce apoptosis independently of RARs (5), whereas $N$-(4-hydoxyphenyl)retinamide can initiate cell death in a receptor-dependent or -independent manner (6). CD437 and MX2870-1 induce apoptosis in the absence of transcription/translation in leukemia cells $(2,7)$. In contrast, up-regulation

Received 11/21/03; revised 6/8/04; accepted 6/14/04.

Grant support: NIH Grants CA 75033 and CA 55681 and California Cancer Research Program Grant 00-00778V-20253 (to F. J. Piedrafita).

The costs of publication of this article were defrayed in part by the payment of page charges. This article must therefore be hereby marked advertisement in accordance with 18 U.S.C. Section 1734 solely to indicate this fact.

Note: Y. Bayon and M. A. Ortiz contributed equally to this work

Requests for reprints: F. Javier Piedrafita, Sidney Kimmel Cancer Center, 10835 Altman Row, San Diego, CA 92121. Phone: (858) 410-4188; Fax: (858) 450-3251; E-mail: jpiedrafita@skcc.org. of several transcription factors [including activator protein (AP)-1, c-myc, and p53] appears to be required for CD437-induced cell death in cell lines derived from solid tumors $(8-10)$.

Clusterin is an enigmatic glycoprotein with a nearly ubiquitous tissue distribution and is apparently involved in a wide range of biological processes (11). Clusterin expression has been associated with tumor formation and prostate cancer progression to androgen independence $(12,13)$. Elevated levels of clusterin have been observed in normal and malignant tissues undergoing apoptosis, including prostate tumor xenografts treated with an inhibitor of ribonucleotide reductase or with the phthalocyanine photosensitizer Pc4 (14, 15). In contrast, an antiapoptotic role has also been suggested (12). Overexpression of clusterin in prostate xenografts models conferred chemoresistance, whereas antisense oligonucleotides or small interfering RNA induced spontaneous apoptosis and increased the anticancer activity of chemotherapeutic agents (16-18). Clusterin is present in several different forms, existing predominantly as a $\sim 70$ $80-\mathrm{kDa}$ dimer that is secreted to extracellular fluids (19). Intracellular clusterin has been documented to exhibit various degrees of glycosylation or maturation (20), and one nuclear form of $\sim 55 \mathrm{kDa}(21)$ has been shown to induce apoptosis $(22,23)$. Clusterin may function as a molecular chaperone $(24,25)$, protecting cells against environmental stresses $(26,27)$.

Whereas a wealth of information has been documented on the induction of apoptosis by CD437 and its analogs, very little is known about the mechanism of MX781 action. We found that this RAR antagonist increased clusterin mRNA and protein expression levels in prostate carcinoma cells. This activity relied on an intact heat shock element (HSE) and AP-1 binding sites in the clusterin promoter and correlated with a strong and sustained activation of heat shock factor (HSF)-1.

\section{MATERIALS AND METHODS}

Cells and Reagents. Prostate carcinoma cells (PC3, DU145, and LNCaP) were obtained from American Type Culture Collection and cultured in RPMI 1640. CV-1 cells were maintained in Dulbecco's modified Eagle's medium. All media contained 10\% fetal bovine serum, 2 mM glutamine, and antibiotics. Synthetic RRMs were obtained from Maxia Pharmaceuticals and Galderma $\mathrm{R} \& \mathrm{D}$, and $10 \mathrm{~mm}$ stock solutions were prepared in DMSO. RRM treatments were performed in medium containing $0.5 \%$ serum. Polyclonal antibodies against clusterin (H-330 and M-18) were acquired from Santa Cruz Biotechnology. Antibodies against HSF-1, Hsp-60, Hsp-70, Hsc-70, and Hsp-90 were from Stressgen. Anti-poly(ADP-ribose) polymerase (PARP) antibody (C2-10) was from BD Biosciences.

Cell proliferation of prostate cancer cells was measured using a 3-(4,5dimethylthiazol-2-yl)-2,5-diphenyltetrazolium bromide colorimetric assay. Cells (3000 cells/well seeded in 96-well plates) were treated (in triplicate points) with increasing concentrations of the RRMs, and the percentage of live cells was measured at different times with respect to control cells grown in the presence of solvent.

Total RNA Extraction and Expression Analysis. Total RNA was isolated using an RNeasy kit (Qiagen). RNase protection assay was performed with the template hAPO-5 (BD Biosciences), as recommended by the manufacturer. Protected RNAs were analyzed by gel electrophoresis using $6 \%$ Tris-borate EDTA-urea polyacrylamide gels and autoradiography. Total RNA $(2.5 \mu \mathrm{g})$ was used to perform reverse transcription using Superscript (Invitrogen). Quantitative real-time PCR assays were performed in triplicate using an ABI 
PRIM 7700 (Applied Biosystems), and quantifications were normalized to an endogenous control [glyceraldehyde-3-phosphate dehydrogenase (GAPDH)]. The primers used to measure clusterin expression were TGTCCCGGCTGGCAAA (forward primer) and AGTGACACCGGAAGGAACGT (reverse primer). A melting curve was used to identify a temperature at which only the amplified fragment, and not primer dimers, accounted for SYBR Green-bound fluorescence.

Transient Transfections and Luciferase Assay. The pGL2 basic luciferase reporter vector containing the rat clusterin 5 '-regulatory region from +57 to -1297 (pRAL) and different deletions has been described previously (28). Mutations were introduced in the pRAL-M/Nsi construct using the QuikChange site-directed mutagenesis kit (Stratagene), and all constructs were confirmed by sequencing. Cells $\left(5 \times 10^{4}\right)$ were seeded in 24 -well plates the day before transfection. CV-1 cells were transfected with $100 \mathrm{ng}$ of the luciferase reporter using a calcium phosphate precipitation procedure for $16 \mathrm{~h}$. Cancer cells were transfected with $500 \mathrm{ng}$ of the reporter using Superfect (LNCaP and PC3) or GenePorter (DU145) transfection reagents for 4-16 h. After transfection, cells were incubated in medium containing $0.5 \%$ serum for $8 \mathrm{~h}$ and then stimulated with drugs for $16 \mathrm{~h}$ before cell lysis. Cell extracts were assayed for firefly luciferase and normalized with protein concentration.

Western Blots. Cells were lysed in 50 mM HEPES (pH 7.4), $100 \mathrm{~mm}$ $\mathrm{NaCl}, 1 \mathrm{~mm}$ EGTA, $1 \%$ Triton X-100, $1 \mathrm{~mm} \mathrm{MgCl}_{2}, 20 \mathrm{~mm} \mathrm{NaF}, 1 \mathrm{~mm}$ sodium vanadate, and a mixture of protease inhibitors (1 mM phenylmethylsulfonyl fluoride, $1 \mu \mathrm{g} / \mathrm{ml}$ aprotinin, and $1 \mu \mathrm{g} / \mathrm{ml}$ leupeptin) or in radioimmunoprecipitation assay buffer $[50 \mathrm{~mm}$ Tris- $\mathrm{HCl}(\mathrm{pH} 7.5), 150 \mathrm{~mm}$ $\mathrm{NaCl}, 1 \% \mathrm{NP} 40,0.5 \%$ sodium deoxycholate, $0.1 \%$ SDS, and protease inhibitors]. Protein extract $(25-50 \mu \mathrm{g})$ was resolved by SDS-PAGE and transferred onto an Immobilon-P membrane (Millipore). The membranes were blocked with Blotto (5\% nonfat milk in Tris-buffered saline containing $0.05 \%$ Tween 20 ) and incubated at $4{ }^{\circ} \mathrm{C}$ for $16-20 \mathrm{~h}$ with the primary antibodies. Incubation with secondary antibodies was subsequently carried out for $1 \mathrm{~h}$ at room temperature. Proteins were detected by enhanced chemiluminescence (ECL; Amersham Pharmacia).

Electrophoretic Mobility Shift Assay. Nuclear extracts were obtained from $10^{6}$ cells following standard procedures. The sequences (in sense orientation) for the oligonucleotides (wild type) containing binding elements from the clusterin promoter were as follows: HSE, GCTTCCAGAAAGCTCCTA; AP-2, ATTCCCCGGCATTCT; AP-1, CTGGCGTGAGTCACG; AP-2/AP-1, ATTCCCCGGCATTCTCTGGCGTGAGTCACG; and CLU, GCTTCCAGAAAGCTCCTAGTGCATTCCCCGGCATTCTCTGGCGTGAGTCACG. Electrophoretic mobility shift assays (EMSAs) were performed basically as described previously (3). Were indicated, samples were incubated with specific antibodies (Active Motif) as recommended by the manufacturer or with an excess of unlabeled oligonucleotides for $30 \mathrm{~min}$ at $4{ }^{\circ} \mathrm{C}$ before the addition of the labeled probe.

Determination of Caspase Activity. DEVDase activity was measured in cell extracts prepared in CE buffer [25 mM PIPES (pH 7), $25 \mathrm{~mm} \mathrm{KCl,} 5 \mathrm{~mm}$ EGTA, $1 \mathrm{~mm}$ DTT, $10 \mu \mathrm{M}$ cytochalasin B, 0.5\% NP40, and a mixture of protease inhibitors]. Ten $\mu \mathrm{g}$ of protein were diluted in $100 \mu \mathrm{l}$ of caspase buffer [50 mм HEPES (pH 7.4), $100 \mathrm{~mm} \mathrm{NaCl}, 1$ mm EDTA, 5 mм DTT, 0.1\% CHAPS, and 10\% sucrose] containing $100 \mu \mathrm{M}$ Acetyl-Asp-Glu-Val-Asp(DEVD)-7-amino-trifluoromethyl coumarin (Enzyme System Products). Reactions were incubated at $37^{\circ} \mathrm{C}$ for $30 \mathrm{~min}$, and the release of free AFC was measured every $2 \mathrm{~min}$ at $510 \mathrm{~nm}$ emission on excitation at $390 \mathrm{~nm}$, using a Victor2 microplate reader (Perkin-Elmer). Slopes were calculated to reflect changes in fluorescence units per minute.

\section{RESULTS}

Retinoid-Related Molecules Inhibit Proliferation and Induce Apoptosis in Prostate Cancer Cells. The antiproliferative activity of synthetic RRMs against prostate carcinoma cells was analyzed by 3-(4,5-dimethylthiazol-2-yl)-2,5-diphenyltetrazolium bromide. Treatment with $1 \mu \mathrm{M}$ of the agonists MX2870-1 and CD2325 or the RAR antagonist MX781 significantly inhibited cell proliferation in a timedependent manner (Fig. 1A). In contrast, little or no effect was observed with all-trans-retinoic acid (RA), which inhibited cell

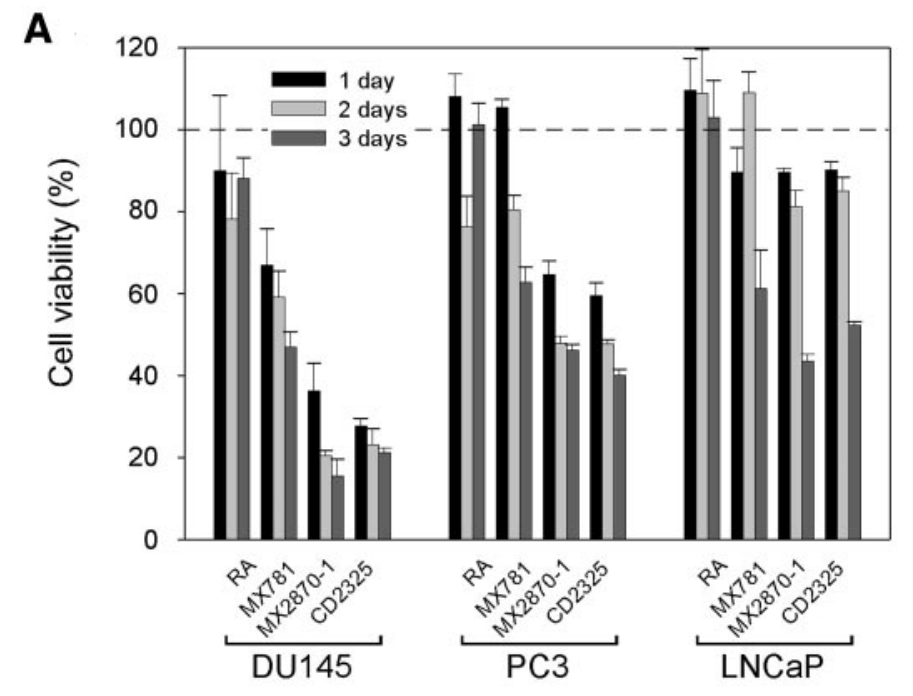

B

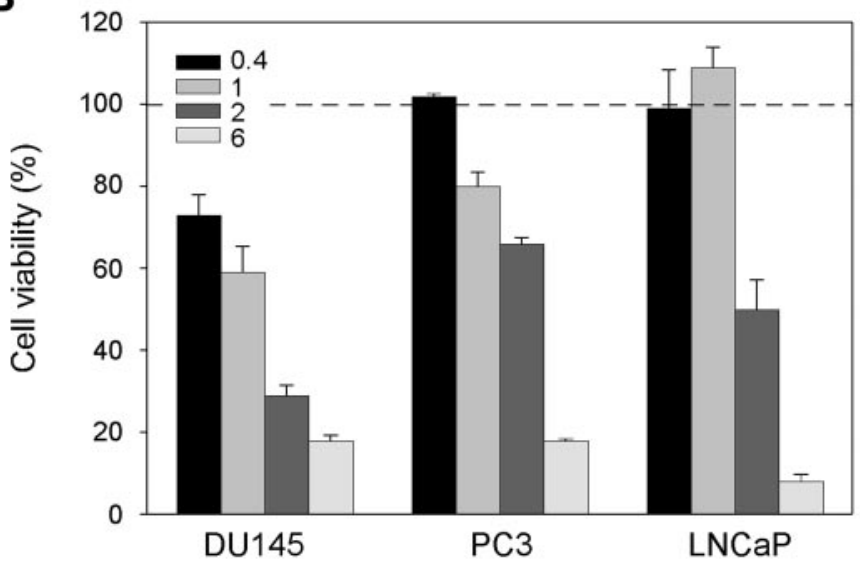

Fig. 1. Selective RRMs inhibit growth of prostate carcinoma cells. RRMs inhibit prostate cancer cell proliferation in a time $(A)$ - and concentration $(B)$-dependent manner. Cells were incubated with $1 \mu \mathrm{M}$ RA or the indicated RRMs for 1,2 , or 3 days $(A)$ or with the indicated concentrations of MX781 (in $\mu \mathrm{M}$ ) for $48 \mathrm{~h}(B)$. The percentage of cell viability was compared with control cells grown in the absence of RRMs. Data show the average $\pm \mathrm{SD}$ of two independent experiments.

growth only after prolonged periods of treatment (data not shown). The growth-inhibitory activity of the synthetic RRMs was enhanced in a dose-dependent manner (Fig. $1 B$; data not shown). This activity correlated with the induction of apoptosis in PC3, DU145, and LNCaP cells, as demonstrated by the induction of caspase activity, DNA fragmentation, and changes in cellular and nuclear morphology (see Ref. 3; data not shown).

MX781 Stimulates the Expression of Clusterin in Prostate Cancer Cells. Using an RNase protection assay, we found that DU145 and LNCaP cells exhibited a robust increase of clusterin mRNA levels on short times of exposure to MX781, which was confirmed by quantitative real-time PCR (Fig. 2A). Induction of clusterin expression was very weak in PC3 cells treated for $4 \mathrm{~h}$, but it increased markedly after prolonged exposure times (Fig. 2B). In contrast, cells treated with RA or other synthetic RAR $\gamma$-selective agonists exhibited no elevated clusterin mRNA levels. The induction of clusterin mRNA levels by MX781 was dependent on the time of exposure and the concentration of RRM (Fig. 2B). Furthermore, we performed immunoblot analysis to demonstrate a significant increase in the protein levels of both full-length (Fig. $2 C$ ) and nuclear (data not shown) forms of clusterin in all three prostate carcinoma cell lines. 
A
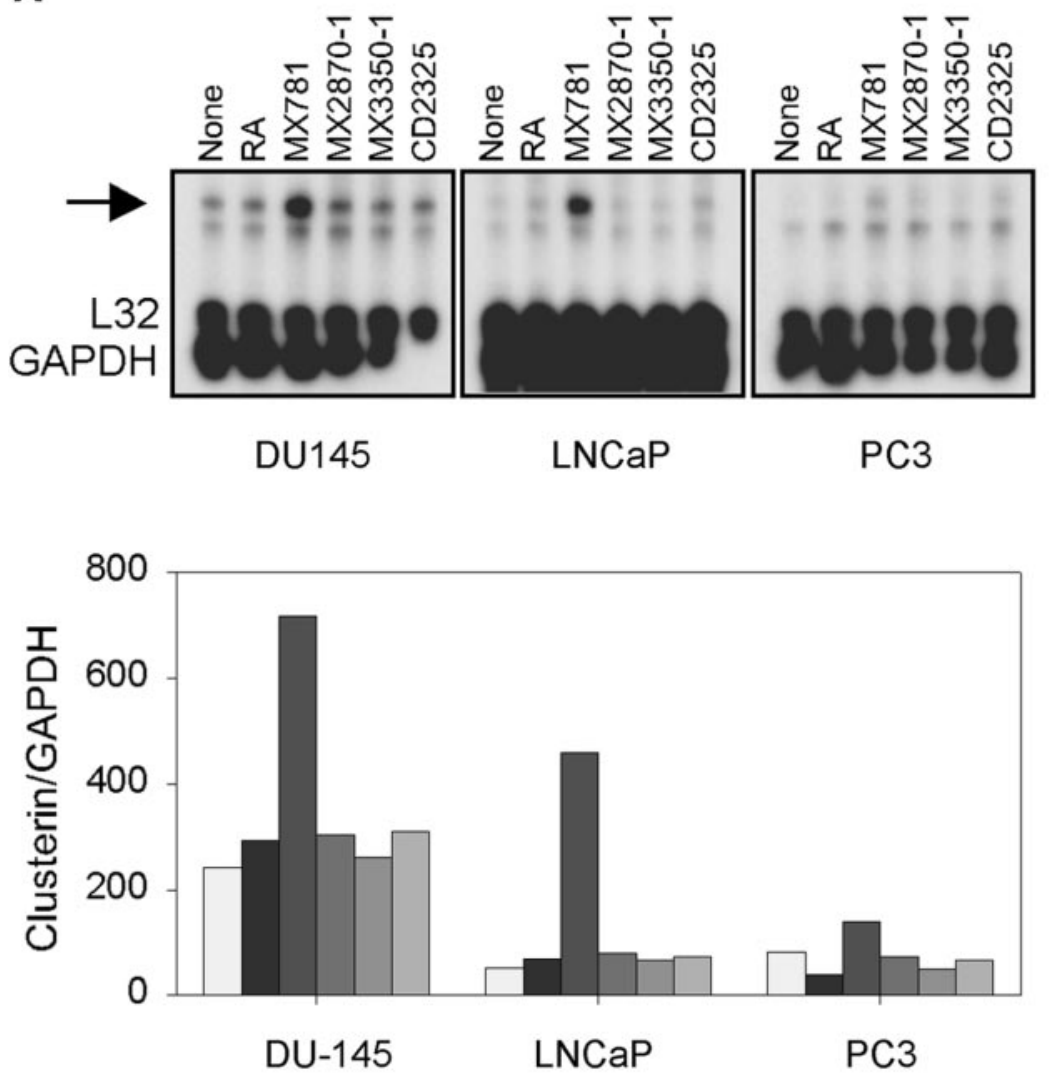

B
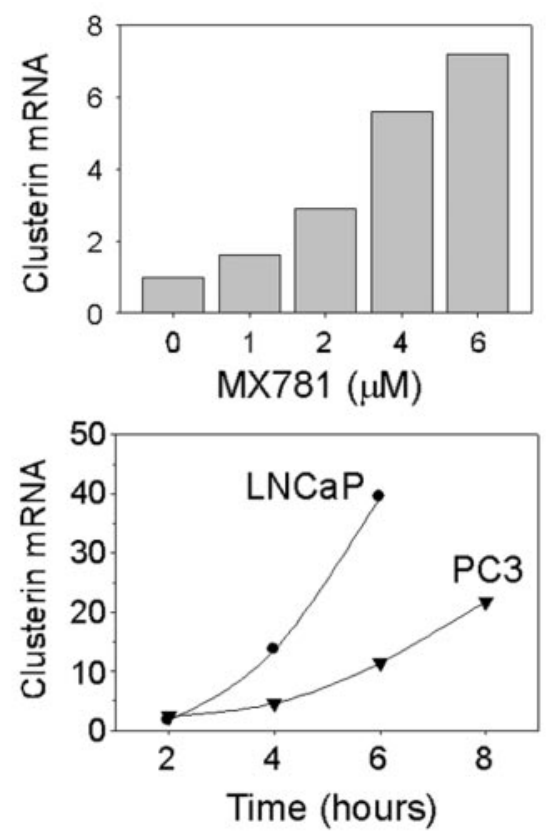

C

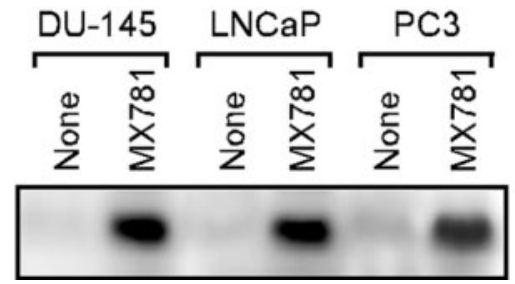

Fig. 2. Induction of clusterin in response to the RRM antagonist MX781. A, induction of clusterin mRNA levels by RRMs. Cells were treated with $6 \mu \mathrm{M}$ of the indicated RRMs,

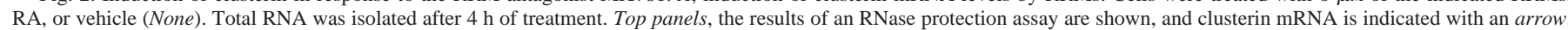

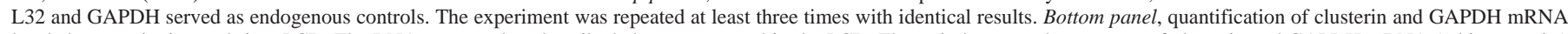

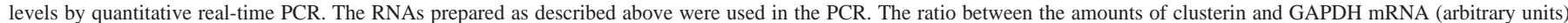

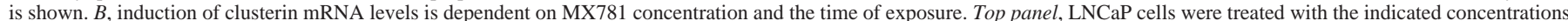

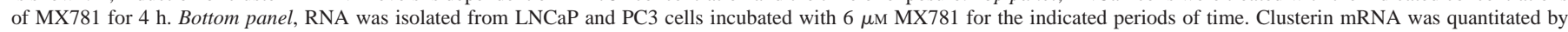

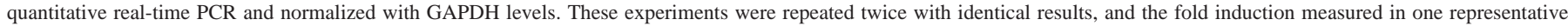

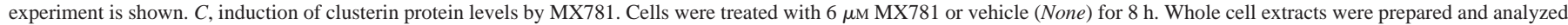
by immunoblot with a clusterin (M-18) antibody. The full-length $(\sim 60 \mathrm{kDa})$ form is shown.

MX781 Induces Transcriptional Activity of the Clusterin Promoter via Heat Shock Element and Activator Protein-1 Sites. To investigate whether MX781-mediated induction of clusterin was a consequence of transcriptional activation of the clusterin promoter, we performed transient transfection experiments in CV-1 cells. We analyzed the $1.3-\mathrm{kb} 5^{\prime}$-promoter region and several deletions of the rat clusterin gene (28). MX781 induced a strong activation (5-fold) of the clusterin promoter, slightly lower than that induced by $12-O$-tetradecanoyl phorbol-13-acetate (TPA). Co-stimulation with RA significantly increased the activation by MX781, whereas RA alone elicited no effect (Fig. 3A). This suggested that transcriptional activation of RARs alone is not sufficient to induce transactivation of the clusterin promoter. Deletion of the proximal region containing HSF-1, AP-2, and AP-1 binding sites completely abrogated MX781-induced activity, whereas TPA was still able to induce luciferase activity (Fig. 3A), probably via distal AP-1 sites (29). We next investigated the effect of other selective RRMs on the clusterin promoter. As observed with RA, various antagonists (CD2366, CD2665, and CD2701) and the RAR $\gamma$-selective agonists MX2870-1 and MX3350-1 were inactive, whereas CD2325 evoked a minimal but reproducible activation (almost 2-fold) of the promoter (Fig. 3B).

Deletion studies identified the region between -30 and -218 , which contains the sites for HSF-1, AP-2, and AP-1, as responsible for MX781-mediated activity. We therefore explored the relative contribution of these sites to the full activation of the clusterin promoter by introducing mutations that abrogate protein binding within the pRALM/Nsi luciferase reporter (Fig. $3 C$ ). Mutation of the HSE site completely abolished promoter activity in DU145 and PC3 cells but partially prevented activation in LNCaP cells (Fig. $3 C$ ). On the other hand, eliminating AP-1 binding partially inhibited clusterin promoter transcriptional activation in LNCaP and DU145 cells, whereas it completely blocked MX781-mediated transactivation in PC3 cells. Mutation of the AP-2 site had no effect. Simultaneous mutation of AP-1 and HSE sites was required to completely abrogate MX781 induction of luciferase activity, indicating that binding of both AP-1 and HSF-1 is needed for the optimal induction of the clusterin promoter. In CV-1 cells, mutating the HSE site completely abrogated the induction of luciferase activity by MX781, whereas mutation of AP-2 or AP-1 sites had no effect (Fig. 3D).

Apoptotic Retinoid-Related Molecules Induce Activator Protein-1 DNA Binding Activity in Prostate Cancer Cells. We then analyzed the effect of MX781 on AP-1 DNA binding activity by EMSA. A substantial DNA binding activity was found using an AP-2/AP-1 oligonucleotide in untreated DU145 cells, which was 907 
A

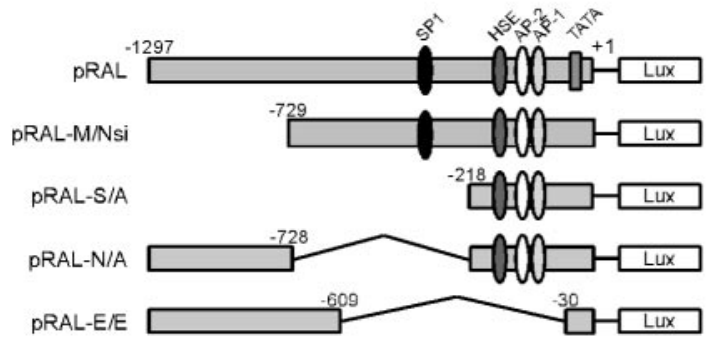

B

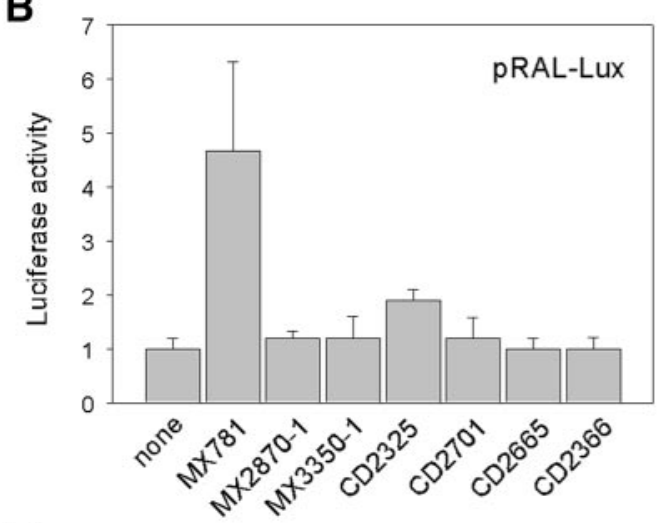

D

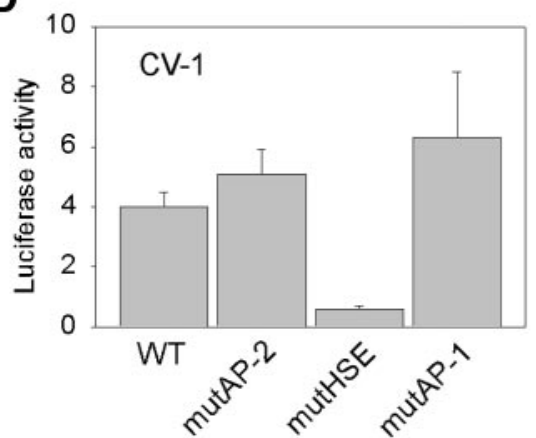

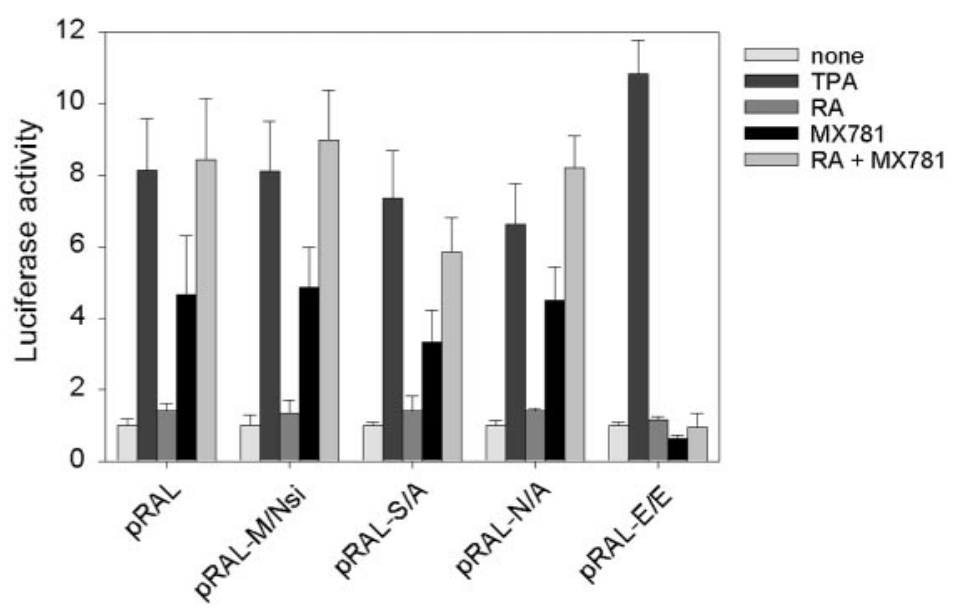
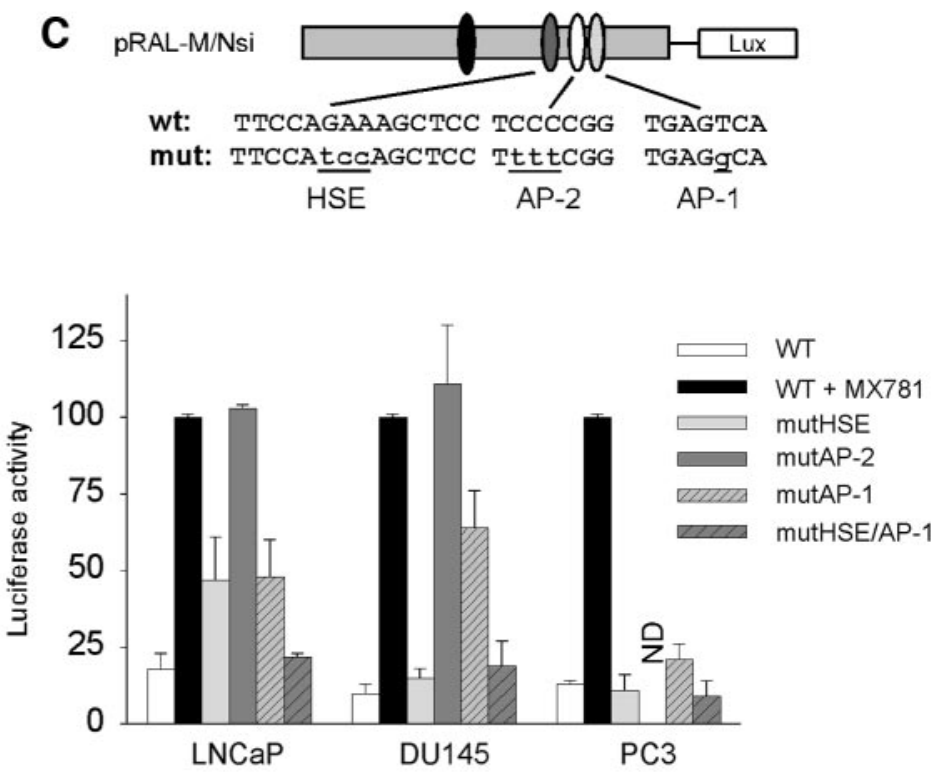

Fig. 3. Deletion and mutational analysis of the rat clusterin promoter region. A, left panel, schematic representation of the rat clusterin promoter and deletion mutants. A, right panel,

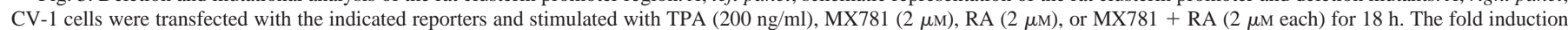

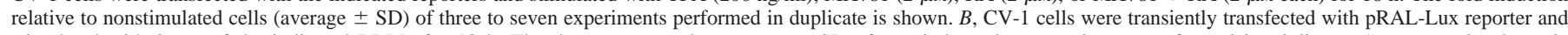

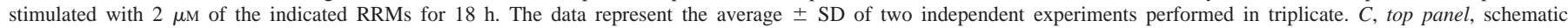

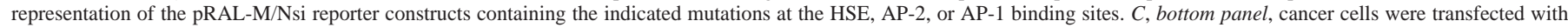

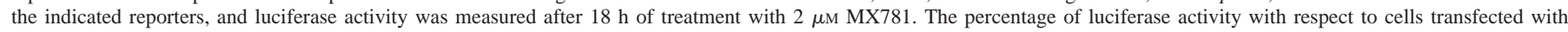

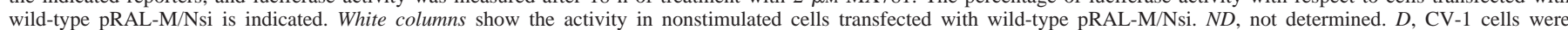

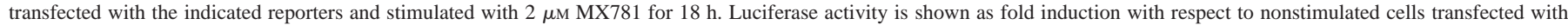
the different constructs. These experiments $(C$ and $D)$ show the average \pm SD from three to six experiments performed in duplicate.

further increased by MX781 exposure (Fig. 4A). The complex was specifically competed with an excess of unlabeled DNA and with an AP-1 oligonucleotide, but not with a DNA containing the AP-2 site alone. Similar binding was observed when the nuclear extracts were incubated with a DNA fragment containing the AP-1 site, whereas no binding to the AP-2 site was observed. Moreover, mutations at the individual binding sites further confirmed the presence of an AP-1 complex, but not AP-2 (Fig. 4A), correlating with the results of the transactivation experiments.

Antibody supershift experiments demonstrated the presence of cJun, JunD, and JunB in the AP-1 complex of resting as well as MX781-treated DU145 cells, whereas antibodies against FosB and CREB-1 did not recognize any protein-DNA complex (Fig. 4B); identical results were observed in LNCaP and PC3 cells and when cells were stimulated with the agonist CD2325 (data not shown). However, cFos was detected only in MX781-treated DU145 cells but not in nonstimulated cells. This same cFos antibody did not supershift the AP-1 complex in extracts prepared from MX781-treated PC3 or LNCaP cells (data not shown). A more substantial increase of AP-1 DNA binding activity was seen in LNCaP cells, but not in PC3 and CV-1 cells; the latter exhibited higher levels of basal AP-1 DNA binding activity as compared with prostate carcinoma cells (Fig. 4C). Together, these data demonstrate a variable induction of AP-1 DNA binding activity by the apoptotic RRMs, despite a strong activation of c-Jun $\mathrm{NH}_{2}$-terminal kinase [JNK (data not shown)].

MX781 but not Agonist Retinoid-Related Molecules Induces Heat Shock Factor-1 DNA Binding Activity. We next investigated protein binding to the HSE located $5^{\prime}$ next to the AP-2/AP-1 site (30). 
Fig. 4. MX781 induces AP-1 DNA binding activity in prostate cancer cells. A, induction of AP-1 DNA binding activity by MX781. DU145 cells were treated with $6 \mu \mathrm{M}$ MX781 or vehicle (control) for $4 \mathrm{~h}$, and then nuclear extracts were prepared and assayed by EMSA using the indicated wild-type or mutated (mut) oligonucleotides. Where indicated, a 50-fold molar excess of unlabeled oligonucleotide (un) was included in the binding reaction. The AP-1 complex is indicated with an arrow. The asterisks indicate nonspecific bands. $B$, antibody supershift of the AP-1 complex. Nuclear extracts were prepared from DU145 cells treated with MX781 or not treated as described above. Binding reactions to a labeled AP-1 oligonucleotide were carried out in the absence (None) or presence of the indicated antibodies before electrophoresis. The specific AP-1 DNA complex is shown (black arrow). The antibody supershifted complexes are indicated by black circles. The open arrow shows the supershift detected exclusively in MX781-stimulated cells with the anti-cFos antibody. $C$, the indicated cells were left untreated (no) or stimulated with $6 \mu \mathrm{M}$ MX781 $(M x)$ for $4 \mathrm{~h}$. Nuclear extracts were assayed for DNA binding activity to the AP-2/AP-1 probe. The specific complex is indicated by an arrow. All of the experiments shown in this figure were carried out two to four times, producing similar outcomes, and one representative result is shown.
A

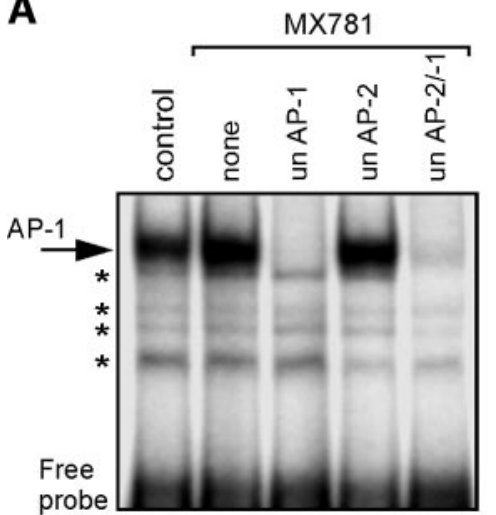

AP-2/AP-1

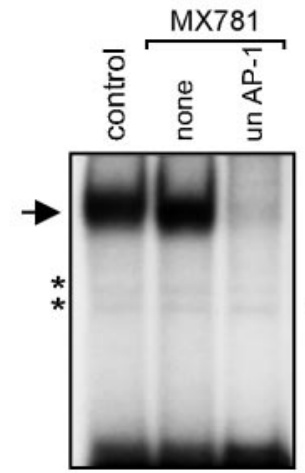

AP-1

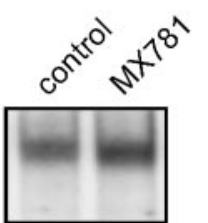

mut AP-2

AP-1

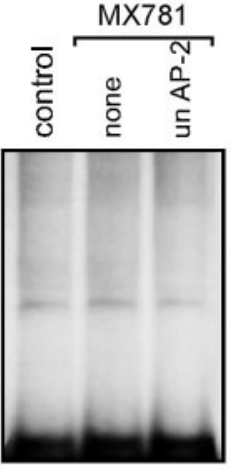

AP-2

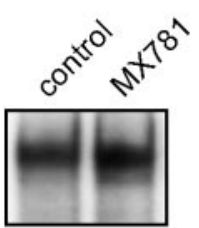

AP-2/AP-1

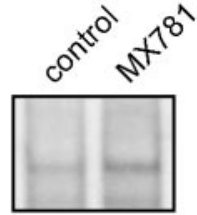

AP-2

mut AP-1
B

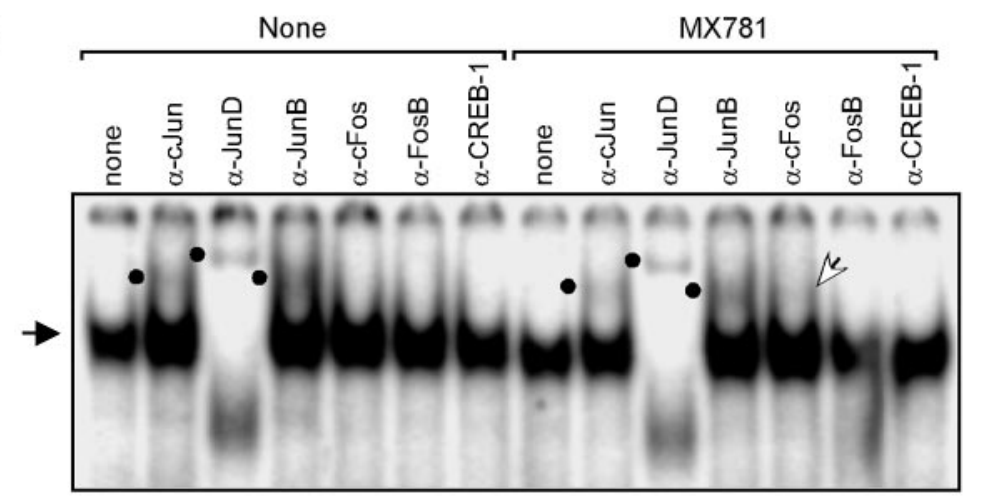

C
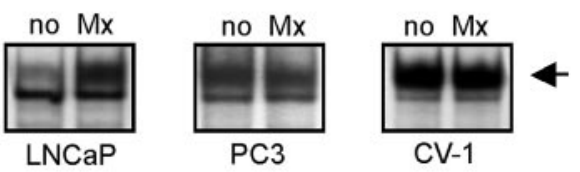

A rapid and strong induction of HSF-1 DNA binding activity was observed in DU145 cells treated with MX781, which was dependent on the time of incubation and the RRM concentration (Fig. 5A). Mutation of the core binding site completely abrogated binding (Fig. $5 B$ ). The protein-DNA complex was totally displaced on incubation with unlabeled wild-type oligonucleotide, but not with mutant DNA. Furthermore, binding was totally abrogated when protein extracts were incubated with anti-HSF-1 antibody, but not with an unrelated antibody (Fig. 5B), proving the presence of HSF-1 in the bound complex. Induction of HSF-1 binding was not observed in cells treated with MX2870-1 or MX3350-1, whereas CD2325 produced a very weak induction of HSF-1 DNA binding (Fig. 5C). Similar results were seen in all prostate cancer cells (data not shown) and correlated with a marginal stimulation of clusterin promoter activity in CV-1 cells (see Fig. 3B). Strong induction of HSF-1 DNA binding by MX781 was also observed in nuclear extracts prepared from LNCaP, PC3, and CV-1 cells. Enhanced HSF-1 activity was not due to increased levels of HSF-1 protein (see below), but it was associated with posttranslational modifications and nuclear translocation of HSF-1 protein, as demonstrated by immunoblot (Fig. 5D).

MX781 Induces a Heat Shock Response in Prostate Carcinoma Cells. Having established that MX781 induces HSF-1 DNA binding and transcriptional activity, we sought to examine the effect of MX781 treatment on certain heat shock proteins (HSPs) at the protein level. As shown in Fig. 6A, all prostate cancer cells treated with MX781, but not with RA, exhibited elevated levels of Hsp-70 and Hsp-90 after 8-24 h of stimulation, concurrent with increased levels of clusterin expression. In contrast, the protein levels of Hsp-60 and Hsc-70 remained unchanged. The agonist CD2325 also enhanced Hsp-70 and Hsp-90 levels after $24 \mathrm{~h}$ of treatment in PC3 and DU145 cells, although to a lesser extent as compared with MX781 (Fig. 6A). This correlated with a weaker induction of HSF-1 DNA binding activity shown earlier. Increased levels of inducible HSPs were associated with posttranslational changes of HSF-1 but not enhanced amounts of HSF-1. Reduced mobility of HSF-1 was 
A

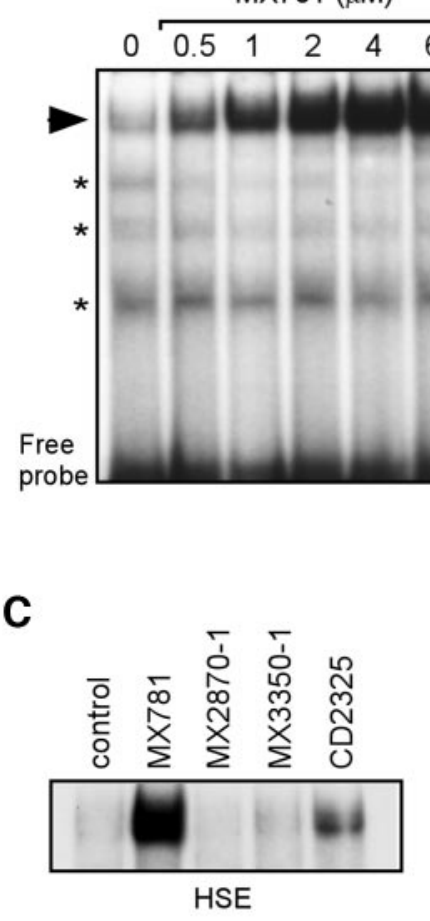

$6 \mu \mathrm{M} M X 781$

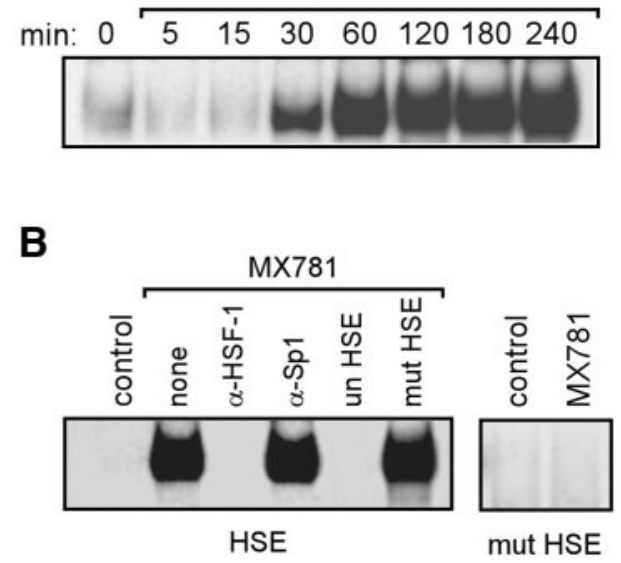

D

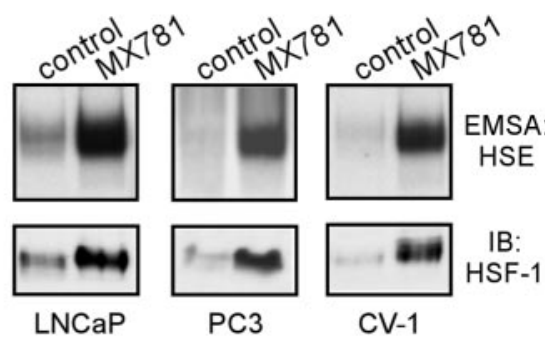

detected in cells treated with MX781 and CD2325 as well (Fig. 6B), probably due to hyperphosphorylation of HSF-1 (31). These changes were seen even after $24 \mathrm{~h}$ of treatment in LNCaP and DU145, indicating a sustained activation of HSF-1.

In PC3 cells, however, treatment with MX781 for extended periods of time ( $24 \mathrm{~h}$ ) caused a complete disappearance of HSF-1 protein (Fig. $6 B$ ). This occurred in parallel with caspase activation, as evidenced by degradation of PARP, a known substrate of caspases. MX781-induced degradation of PARP and HSF-1 was weaker in LNCaP and DU145 cells, suggesting a delay in the induction of apoptosis. To demonstrate that HSF-1 degradation was indeed a consequence of caspase activity, we incubated cells with the pan-caspase inhibitor z-Val-Ala-Aspfluoromethyl ketone (z-VAD-fmk) before MX781 stimulation. This inhibitor efficiently blocked the induction of DEVDase activity (Fig. $6 C$ ) and prevented the degradation of HSF-1 and PARP (Fig. 6D) induced by MX781. In contrast, z-VAD-fmk elicited no effect on Hsp70 protein levels, indicating that heat shock response was independent of caspase activity.

\section{DISCUSSION}

Complex signaling pathways are activated in response to cellular stress, which result in the activation of key transcription factors and the rapid synthesis of proteins that function to induce apoptosis or to repair and protect cells from dying. Certain pathways, such as JNK/ AP-1 and $\mathrm{I} \kappa \mathrm{B}$ kinase (IKK)/nuclear factor $\kappa \mathrm{B}$, can signal survival or apoptosis decisions in a cell type, context, and stimulus-dependent manner (32). Synthetic RRMs with strong anticancer activity have been shown to activate (JNK) or inhibit (IKK) these pathways, which correlates with their ability to induce apoptosis $(3,33)$. Another signaling pathway that responds to cellular stress is the heat shock response, which results in the rapid synthesis of HSPs that function as molecular chaperones or proteases to repair damaged proteins or degrade them (34). Enhanced heat shock gene expression is regulated by the four-member HSF family of transcription factors (35), with
HSF-1 playing a major role in the cellular response to thermal stress (36). A large body of evidence supports a protective role of HSPs against cellular stress (37). Hsp-70 inhibits apoptosis downstream of cytochrome $c$ release, perhaps by preventing recruitment of procaspase 9 to the apoptosome and interfering with apoptosis-inducing factor $(38-40)$. However, strong and prolonged activation of heat shock response can also be detrimental and cause cell death (41), and a proapoptotic activity has been suggested for Hsp-60 and Hsp-90 (42).

In addition to elevated temperatures, heat shock response is activated by several cytotoxic chemicals, including hydrogen peroxide, antiproliferative prostaglandins, and sodium arsenite (43-45). These compounds can induce apoptosis, although a direct connection between the activation of heat shock and the induction of apoptosis needs to be established. We now report on a novel RAR antagonist, MX781, which induces a strong activation of HSF-1 and causes apoptosis in vitro and in vivo $(3,46)$. MX781 and the RAR $\gamma$ agonists MX2870-1 and CD2325 share structural similarities (including an adamantyl group and a carboxylic moiety) and induce cell death in a caspase-dependent manner. However, agonist RRMs are more potent inducers of apoptosis than the antagonist MX781, which usually requires higher concentrations and reduced amounts of serum in vitro (47). In addition to exhibiting opposite RAR-dependent activities, the mechanism of apoptosis induction of agonist RRMs and antagonist MX781 also seems quite distinct (48). Besides activating JNK and p38 stress kinases and inhibiting IKK $(3,47)$, MX781 also enhances HSF-1 transcriptional activity. The lack of heat shock stress in response to RA and other agonist RRMs suggests that this novel activity of MX781 is probably independent of RAR-mediated transcriptional activity. This is further suggested by the fact that co-stimulation with RA enhances rather than inhibits MX781-mediated transactivation of the clusterin promoter.

The induction of HSF-1 DNA binding and transcriptional activity by MX781 is very strong and lasts for extended periods of time. 
A

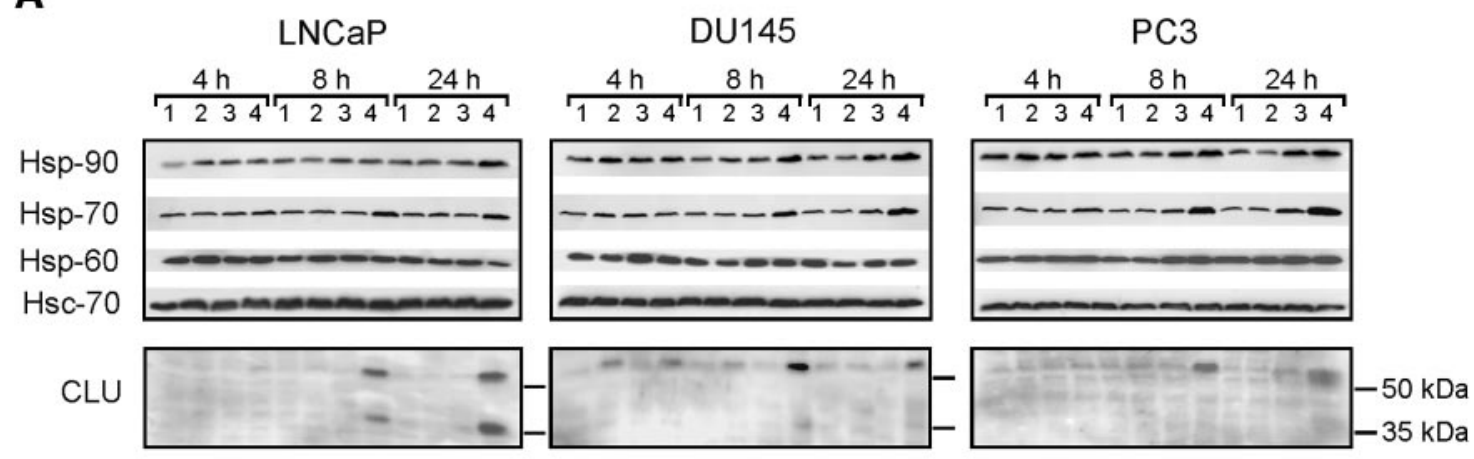

B

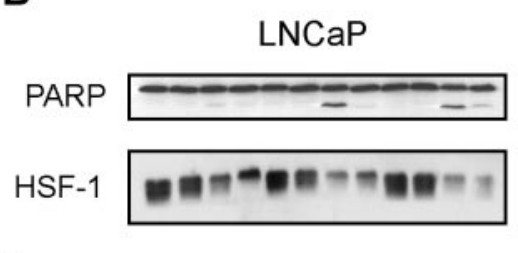

C

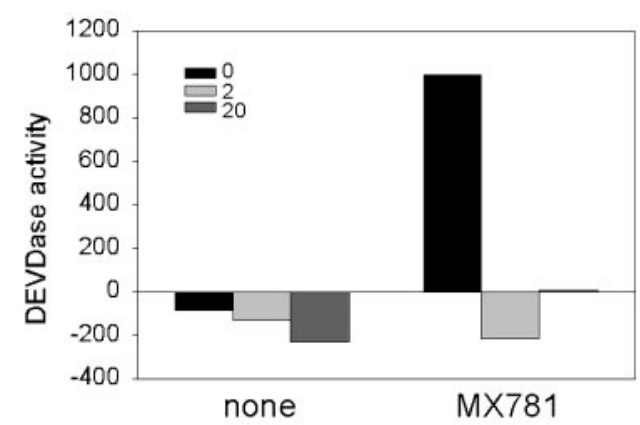

DU145

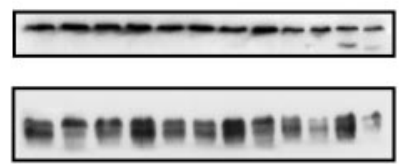

D

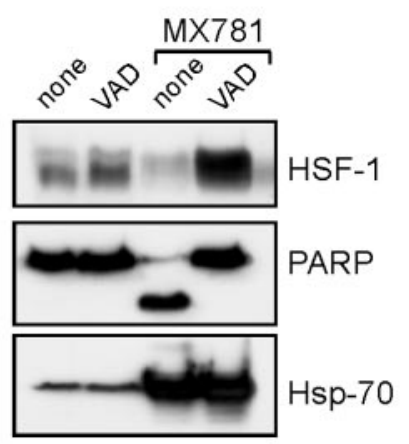

Fig. 6. Induction of HSP expression and apoptosis by MX781 in prostate cancer cells. A, cells were incubated with vehicle (Lanes 1) or 6 4M RA (Lanes 2), CD2325 (Lanes 3),

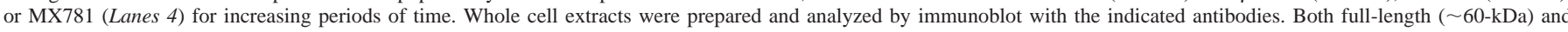

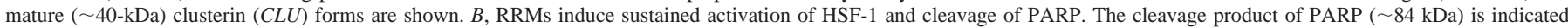

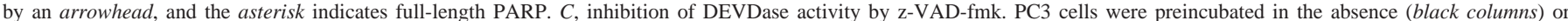

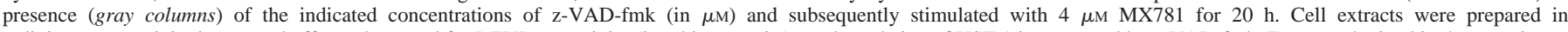

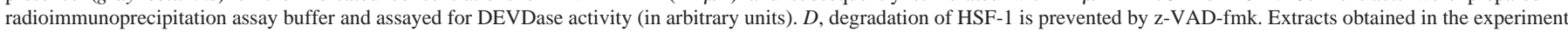
shown before were resolved by SDS-PAGE and analyzed by immunoblot with the indicated antibodies.

Whether or not the activation of HSF-1 by MX781 affects the induction of apoptosis by the RRM is currently under investigation, but preliminary studies have shown that the presence of HSF-1 does indeed enhance MX781-induced apoptosis. ${ }^{3}$ This correlates with other studies linking the overexpression of hyperactivated HSF-1 with cell death (41). An additional link between the activation of a heat shock response and apoptosis is the increase in clusterin mRNA and protein levels observed in MX781-stimulated cells. Maximum increase of clusterin levels relies on the presence of an intact HSE, although AP-1 transcription factors may also play a role in prostate carcinoma cells in a cell type-specific manner. Interestingly, the highest induction of clusterin mRNA expression observed in $\mathrm{LNCaP}$ cells coincided with the highest induction of AP-1 DNA binding activity. On the contrary, the induction of mRNA clusterin expression was considerably delayed in PC3 cells, which exhibit high levels of basal AP-1 DNA binding activity that are minimally affected by MX781 stimulation. Independently of the actual role of AP-1 activity or associated cofactors on MX781 function, activation of the JNK/AP-1 pathway alone is not sufficient for the induction of clusterin expression because the agonist

${ }^{3}$ F. Javier Piedrafita, Y. Bayon, and M. A. Ortiz. Involvement of HSF-1 in MX781induced apoptosis, manuscript in preparation.
RRMs CD2325 and MX2870-1 had minimal effects on clusterin expression, despite a strong activation of JNK. Therefore, optimal induction of clusterin promoter activity relies on a complex interplay between HSF-1 and AP-1 transcription factors and probably additional cell type-specific cofactors as well.

Although clusterin was originally thought to mediate apoptosis (49) and has been used as an apoptotic marker, many studies have suggested an antiapoptotic function $(26,27)$, which is more in agreement with its hypothetical chaperone activity. Nevertheless, different functions can be attributed to the various cellular forms of clusterin. Overexpression of a nuclear truncated form of clusterin has been shown to cause apoptosis $(22,23)$. On ionizing radiation or transforming growth factor $\beta$ stimulation, levels of truncated clusterin increase, and the protein undergoes posttranslational modifications (perhaps glycosylation) and translocation into the nucleus (21), where it can bind $\mathrm{Ku} 70$ protein and induce apoptosis independently of caspases (22). Thus, although the induction of full-length clusterin seems to have a cytoprotective effect, concurrent generation of the truncated nuclear form may lead to apoptosis in cell type- and stimulus-dependent manners. High levels of stress by continuous exposure to MX781 could turn the cytoprotective activity of clusterin 5911 
and certain HSPs (such as Hsp-70) into the proapoptotic activity of nuclear clusterin and/or other HSPs. Whether clusterin actively participates in MX781-induced apoptosis is being explored.

In conclusion, we have reported on a novel function of the RRM antagonist MX781 in prostate cancer cells, which is the activation of HSF-1 pathway and subsequent induction of clusterin mRNA and protein levels. Binding of HSF-1 is essential for the optimal activation of the clusterin promoter and cooperates with AP-1 for the full induction of clusterin expression by MX781. The involvement of HSF-1/AP-1/clusterin in MX781-induced apoptosis would argue in favor of a transcription/translation-dependent mechanism of action, as observed with CD437 and other agonist RRMs in cell lines derived from solid tumors.

\section{ACKNOWLEDGMENTS}

We thank Renata Hasim for excellent technical assistance; Magnus Pfahl, Maxia Pharmaceuticals, and Galderma R\&D for the synthetic retinoids; and Marion Sauter for secretarial assistance.

\section{REFERENCES}

1. Piedrafita FJ, Pfahl M. Nuclear retinoid receptors and mechanism of action. In: Nau H, Blaner WS, editors. Retinoids: the biochemical and molecular basis of vitamin A and retinoid action. Berlin: Springer-Verlag; 1999. p. 153-84.

2. Piedrafita FJ, Pfahl M. Retinoid-induced apoptosis and Sp1 cleavage occur independently of transcription and require caspase activation. Mol Cell Biol 1997; 17:6348-58.

3. Bayon Y, Ortiz MA, Lopez-Hernandez FJ, et al. Inhibition of $\mathrm{I} \kappa \mathrm{B}$ kinase by a new class of retinoid-related anticancer agents that induce apoptosis. Moll Cell Biol 2003;23:1061-74.

4. Hsu CA, Rishi AK, Su-Li X, et al. Retinoid induced apoptosis in leukemia cells through a retinoic acid nuclear receptor-independent pathway. Blood 1997; 89:4470-9.

5. Chun KH, Benbrook DM, Berlin KD, Hong WK, Lotan R. The synthetic heteroarotinoid SHetA2 induces apoptosis in squamous carcinoma cells through a receptorindependent and mitochondria-dependent pathway. Cancer Res 2003;63:3826-32.

6. Sun SY, Li W, Yue P, et al. Mediation of N-(4-hydoxyphenyl)retinamide-induced apoptosis in human cancer cells by different mechanisms. Cancer Res 1999;59: 2493-8.

7. Mologni L, Ponzanelli I, Bresciani F, et al. The novel synthetic retinoid 6-[3adamantyl-4-hydroxyphenyl]-2-naphthalene carboxylic acid (CD437) causes apoptosis in acute promyelocytic leukemia cells through rapid activation of caspases. Blood 1999; $93: 1045-61$.

8. Schadendorf D, Kern MA, Artuc M, et al. Treatment of melanoma cells with the synthetic retinoid CD437 induces apoptosis via activation of AP-1 in vitro, and causes growth inhibition in xenografts in vivo. J Cell Biol 1996;135:1889-98.

9. Sun SY, Yue P, Shroot B, Hong WK, Lotan R. Implication of c-Myc in apoptosis induced by the retinoid CD437 in human lung carcinoma cells. Oncogene 1999;18: 3894-901.

10. Sun SY, Yue P, Wu GS, et al. Implication of p53 in growth arrest and apoptosis induced by the synthetic retinoid CD437 in human lung cancer cells. Cancer Res 1999;59:2829-33.

11. Trougakos IP, Gonos ES. Clusterin/apolipoprotein $\mathrm{J}$ in human aging and cancer. Int J.Biochem Cell Biol 2002;34:1430-48.

12. Miyake H, Nelson C, Rennie PS, Gleave ME. Testosterone-repressed prostate message- 2 is an antiapoptotic gene involved in progression to androgen independence in prostate cancer. Cancer Res 2000;60:170-6.

13. Ouyang XS, Wang X, Lee DT, Tsao SW, Wong YC. Up-regulation of TRPM-2, MMP-7 and ID-1 during sex hormone-induced prostate carcinogenesis in the Noble rat. Carcinogenesis (Lond) 2001;22:965-73.

14. Wright PS, Cross-Doersen D, Th'ng JP, et al. A ribonucleotide reductase inhibitor, MDL 101,731, induces apoptosis and elevates TRPM-2 mRNA levels in human prostate tumor xenografts. Exp Cell Res 1996;222:54-60.

15. Kalka K, Ahmad N, Criswell T, Boothman D, Mukhtar H. Up-regulation of clusterin during phthalocyanine 4 photodynamic therapy-mediated apoptosis of tumor cells and ablation of mouse skin tumors. Cancer Res 2000;60:5984-7.

16. Miyake H, Nelson C, Rennie PS, Gleave ME. Acquisition of chemoresistant phenotype by overexpression of the antiapoptotic gene testosterone-repressed prostate message-2 in prostate cancer xenograft models. Cancer Res 2000;60:2547-54.

17. Miyake H, Chi KN, Gleave ME. Antisense TRPM-2 oligodeoxynucleotides chemosensitize human androgen-independent PC-3 prostate cancer cells both in vitro and in vivo. Clin Cancer Res 2000;6:1655-63.

18. Trougakos IP, So A, Jansen B, Gleave ME, Gonos ES. Silencing expression of the clusterin/apolipoprotein $\mathbf{J}$ gene in human cancer cells using small interfering RNA induces spontaneous apoptosis, reduced growth ability, and cell sensitization to genotoxic and oxidative stress. Cancer Res 2004;64:1834-42.
19. Wong P, Taillefer D, Lakins J, et al. Molecular characterization of human TRPM-2/ clusterin, a gene associated with sperm maturation, apoptosis and neurodegeneration. Eur J Biochem 1994;221:917-25.

20. Lakins J, Bennett SA, Chen JH, et al. Clusterin biogenesis is altered during apoptosis in the regressing rat ventral prostate. J Biol Chem 1998;273:27887-95.

21. Reddy KB, Jin G, Karode MC, Harmony JA, Howe PH. Transforming growth factor beta (TGF beta)-induced nuclear localization of apolipoprotein J/clusterin in epithelial cells. Biochemistry 1996;35:6157-63.

22. Yang CR, Leskov K, Hosley-Eberlein K, et al. Nuclear clusterin/XIP8, an X-rayinduced Ku70-binding protein that signals cell death. Proc Natl Acad Sci USA 2000;97:5907-12

23. Leskov KS, Klokov DY, Li J, Kinsella TJ, Boothman DA. Synthesis and functiona analyses of nuclear clusterin, a cell death protein. J Biol Chem 2003;278:11590-600.

24. Humphreys DT, Carver JA, Easterbrook-Smith SB, Wilson MR. Clusterin has chaperone-like activity similar to that of small heat shock proteins. J Biol Chem 1999; 274:6875-81.

25. Poon S, Easterbrook-Smith SB, Rybchyn MS, Carver JA, Wilson MR. Clusterin is an ATP-independent chaperone with very broad substrate specificity that stabilizes stressed proteins in a folding-competent state. Biochemistry 2000;39:15953-60.

26. Sensibar JA, Sutkowski DM, Raffo A, et al. Prevention of cell death induced by tumor necrosis factor alpha in LNCaP cells by overexpression of sulfated glycoprotein-2 (clusterin). Cancer Res 1995;55:2431-7.

27. Humphreys D, Hochgrebe TT, Easterbrook-Smith SB, Tenniswood MP, Wilson MR Effects of clusterin overexpression on TNFalpha- and TGFbeta-mediated death of L929 cells. Biochemistry 1997;36:15233-43.

28. Jin G, Howe PH. Regulation of clusterin gene expression by transforming growth factor beta. J Biol Chem 1997;272:26620-6.

29. Wong P, Pineault J, Lakins J, et al. Genomic organization and expression of the rat TRPM-2 (clusterin) gene, a gene implicated in apoptosis. J Biol Chem 1993;268: 5021-31.

30. Michel D, Chatelain G, North S, Brun G. Stress-induced transcription of the clusterin/ apoJ gene. Biochem J 1997;328:45-50.

31. Holmberg CI, Hietakangas V, Mikhailov A, et al. Phosphorylation of serine 230 promotes inducible transcriptional activity of heat shock factor 1. EMBO J 2001;20: $3800-10$.

32. Herr I, Debatin KM. Cellular stress response and apoptosis in cancer therapy. Blood 2001;98:2603-14

33. Ortiz MA, Lopez-Hernandez FJ, Bayon Y, Pfahl M, Piedrafita FJ. Retinoid-related molecules induce cytochrome $\mathrm{c}$ release and apoptosis through activation of c-Jun $\mathrm{NH}_{2}$-terminal kinase/p38 mitogen-activated protein kinases. Cancer Res 2001;61: $8504-12$.

34. Morimoto RI. Regulation of the heat shock transcriptional response: cross talk between a family of heat shock factors, molecular chaperones, and negative regulators. Genes Dev 1998;12:3788-96.

35. Wu C. Heat shock transcription factors: structure and regulation. Annu Rev Cell Dev Biol 1995; 11:441-69.

36. McMillan DR, Xiao X, Shao L, Graves K, Benjamin IJ. Targeted disruption of hea shock transcription factor 1 abolishes thermotolerance and protection against heatinducible apoptosis. J Biol Chem 1998;273:7523-8.

37. Jaattela M. Escaping cell death: survival proteins in cancer. Exp Cell Res 1999;248: $30-43$.

38. Li CY, Lee JS, Ko YG, Kim JI, Seo JS. Heat shock protein 70 inhibits apoptosis downstream of cytochrome c release and upstream of caspase-3 activation. J Biol Chem 2000;275:25665-71.

39. Beere HM, Wolf BB, Cain K, et al. Heat-shock protein 70 inhibits apoptosis by preventing recruitment of procaspase- 9 to the Apaf-1 apoptosome. Nat Cell Biol 2000;2:469-75

40. Ravagnan L, Gurbuxani S, Susin SA, et al. Heat-shock protein 70 antagonizes apoptosis-inducing factor. Nat Cell Biol 2001;3:839-43.

41. Nakai A, Suzuki M, Tanabe M. Arrest of spermatogenesis in mice expressing an active heat shock transcription factor 1. EMBO J 2000;19:1545-54.

42. Garrido C, Gurbuxani S, Ravagnan L, Kroemer G. Heat shock proteins: endogenous modulators of apoptotic cell death. Biochem Biophys Res Commun 2001;286:43342.

43. Amici C, Sistonen L, Santoro MG, Morimoto RI. Antiproliferative prostaglandins activate heat shock transcription factor. Proc Natl Acad Sci USA 1992;89:6227-31.

44. Jacquier-Sarlin MR, Polla BS. Dual regulation of heat-shock transcription factor (HSF) activation and DNA-binding activity by $\mathrm{H}_{2} \mathrm{O}_{2}$ : role of thioredoxin. Biochem $\mathrm{J}$ 1996;318:187-93

45. Zhong M, Orosz A, Wu C. Direct sensing of heat and oxidation by Drosophila heat shock transcription factor. Mol Cell 1998;2:101-8.

46. Fanjul AN, Piedrafita FJ, Al-Shamma H, Pfahl M. Apoptosis induction and potent antiestrogen receptor-negative breast cancer activity in vivo by a retinoid antagonist Cancer Res 1998;58:4607-10.

47. Lopez-Hernandez FJ, Ortiz MA, Bayon Y, Piedrafita FJ. Reduced concentrations of serum enhance the antiproliferative activity of retinoid-related molecules and accelerate the onset of apoptosis. Biochem Pharmacol 2003;65:2021-30.

48. Lopez-Hernandez FJ, Ortiz MA, Bayon Y, Piedrafita FJ. Retinoid-related molecules require caspase 9 for the effective release of Smac and the rapid induction of apoptosis. Cell Death Differ 2004;11:154-64.

49. Rosenberg ME, Silkensen J. Clusterin: physiologic and pathophysiologic considerations. Int J Biochem Cell Biol 1995;27:633-45. 


\section{Cancer Research}

\section{The Retinoid Antagonist MX781 Induces Clusterin Expression in Prostate Cancer Cells via Heat Shock Factor-1 and Activator Protein-1 Transcription Factors}

Yolanda Bayon, Maria A. Ortiz, Francisco J. Lopez-Hernandez, et al.

Cancer Res 2004;64:5905-5912.

Updated version Access the most recent version of this article at:

http://cancerres.aacrjournals.org/content/64/16/5905

Cited articles This article cites 48 articles, 31 of which you can access for free at:

http://cancerres.aacrjournals.org/content/64/16/5905.full\#ref-list-1

Citing articles This article has been cited by 4 HighWire-hosted articles. Access the articles at:

http://cancerres.aacrjournals.org/content/64/16/5905.full\#related-urls

E-mail alerts Sign up to receive free email-alerts related to this article or journal.

Reprints and To order reprints of this article or to subscribe to the journal, contact the AACR Publications Subscriptions Department at pubs@aacr.org.

Permissions To request permission to re-use all or part of this article, use this link http://cancerres.aacrjournals.org/content/64/16/5905.

Click on "Request Permissions" which will take you to the Copyright Clearance Center's (CCC)

Rightslink site. 